\title{
Neural Networks Applied to the Wave-Induced Fatigue Analysis of Steel Risers
}

\author{
João P. R. Cortina (iD, Fernando J. M. de Sousa, and Luis V. S. Sagrilo \\ Laboratory of Analysis and Reliability of Offshore Structures, Civil Engineering Program/COPPE, Federal University of Rio de Janeiro, \\ Rio de Janeiro, Brazil \\ Correspondence should be addressed to João P. R. Cortina; jpr.cortina@gmail.com
}

Received 12 March 2018; Revised 20 May 2018; Accepted 6 June 2018; Published 25 July 2018

Academic Editor: Lotfi Senhadji

Copyright (C) 2018 João P. R. Cortina et al. This is an open access article distributed under the Creative Commons Attribution License, which permits unrestricted use, distribution, and reproduction in any medium, provided the original work is properly cited.

\begin{abstract}
Time domain stochastic wave dynamic analyses of offshore structures are computationally expensive. Considering the waveinduced fatigue assessment for such structures, the combination of many environmental loading cases and the need of long timeseries responses make the computational cost even more critical. In order to reduce the computational burden related to the wave-induced fatigue analysis of Steel Catenary Risers (SCRs), this work presents the application of a recently developed hybrid methodology that combines dynamic Finite Element Analysis (FEA) and Artificial Neural Networks (ANN). The methodology is named hybrid once it requires short time series of structure responses (obtained by FEA) and imposed motions (evaluated analytically) to train an ANN. Subsequently, the ANN is employed to predict the remaining response time series using the prescribed motions imposed at the top of the structure by the floater unit. In this particular work, the methodology is applied aiming to predict the tension and bending moments' time series at structural elements located at the top region and at the touchdown zone (TDZ) of a metallic riser. With the predicted responses (tensions and moments), the stress time series are determined for eight points along the pipe cross sections, and stress cycles are identified using a Rainflow algorithm. Fatigue damage is then evaluated using SN curves and the Miner-Palmgren damage accumulation rule. The methodology is applied to a SCR connected to a semisubmersible platform in a water depth of $910 \mathrm{~m}$. The obtained results are compared to those from a full FEA in order to evaluate the accuracy and computer efficiency of the hybrid methodology.
\end{abstract}

\section{Introduction}

As the oil industry faces the challenge of production on harsh environments, the design of offshore structures requires progressively a better understanding of the environment and the use of more sophisticated numerical methods, capable of representing the nonlinearities involved in the dynamic analysis of such structures. Both requirements, however, can lead to very high computational costs.

This is a consequence of the combination of refined numerical models and the need of several long stochastic time domain simulations that are needed to assure reliable statistics for the response parameters investigated. This aspect can be crucial in the wave-induced fatigue analysis of risers or in early design stages, where many structural configurations should be analyzed.
Depending on the water depth, geometric configuration, finite element mesh refinement, use of nonlinear physical properties, and nonlinear beam element formulation, a single 3-hour FEM-based stochastic dynamic analysis of a SCR can demand more than 3 hours of computational processing in a modern desktop computer. As, in the design practice, the wave-induced riser fatigue analysis requires the stochastic dynamic analysis for many load cases (sea state conditions), the computer burden of this analysis is tremendous.

One of the most promising alternatives to evaluate dynamic responses of marine structures with a significant reduction in computational time is the utilization of hybrid methods combining FEA with Artificial Neural Networks (ANNs) [1-7]. The basic idea of these methods is to employ the remarkable capacity of learning, generalization, and prediction of neural networks to replace the onerous numerical 


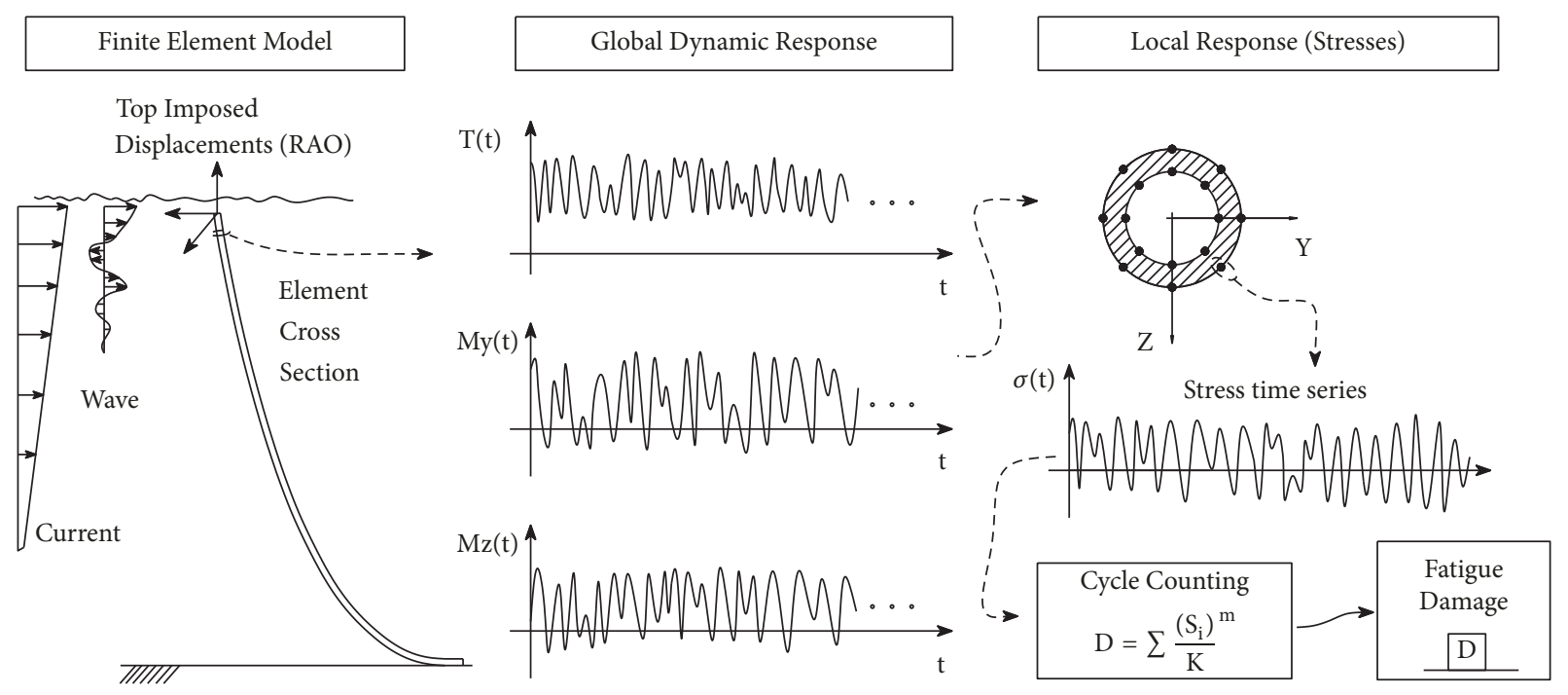

FIGURE 1: Steel riser fatigue procedure.

integration of a time domain dynamic analysis by finite elements method. In the most usual application, short time series of motions (imposed at the top of the structure by the floater unit) and the corresponding induced structure responses (provided by the FEA) can be used to train an ANN. Then, once the floater motions can be evaluated analytically, the remaining of the response time series is predicted by the ANN.

Guarize et al. [1] applied the hybrid ANN-FEA methodology in the prediction of the dynamic top tension of a mooring line and in the evaluation of the DNV-LRFD utilization factor [8] in the sagbend zone of a SLWR (Steel Lazy-Wave Riser). Both structures were connected to a FPSO (Floating, Production, Storage, and Offloading unit). The predictions showed very good agreement with the FEA results, and the computational time was reduced by a factor of about 20 times. Pina et al. [2] presented hybrid methods which use dynamic autoregressive models applied to the prediction of risers and mooring lines' top tensions. Christiansen et al. $[3,4]$ used a similar hybrid methodology in the prediction of top tensions for the fatigue assessment of a mooring line with a substantial reduction in the total simulation time. Chaves et al. [5] studied the application of the hybrid method in the prediction of tension and curvatures in the bend stiffener region of a flexible riser and used these results to perform a fatigue verification. Kim [6, 7] employed a NARX-based Volterra model for the prediction of dynamic response of slender marine structures, also obtaining good results.

This work presents an application of a hybrid ANNFEA method for the prediction of axial tension and bending moments at the top region and at the touchdown zone of a SCR connected to a semisubmersible platform, in order to reduce computational costs of time domain stochastic simulations. To the authors' knowledge, the previous works did not employ a similar approach to predict the load effects for riser points near the TDZ nor to evaluate the total fatigue damage in this region.
The methodology is then used for all load cases (sea states) in order to evaluate fatigue damage in these critical regions. The fatigue results and also the computational costs of the hybrid method and those of the usual FEA approach are compared.

\section{Stochastic Time Domain Fatigue Assessment of Metallic Risers}

Steel risers are structures composed of welded segments of conventional steel pipes [9] and used for oil or gas production and for gas exportation. The most common configurations are the free hanging catenary (SCR) and the lazy-wave (SLWR, Steel Lazy-Wave Riser).

The wave-induced fatigue analysis of such structures is traditionally divided into two steps: the global and the local analysis. In the global analysis, a nonlinear FE-based time domain stochastic dynamic simulation is employed for each loading case to get the corresponding time series of axial tensions and bending moments along the riser. Usually, the global analysis is performed using a decoupled procedure, where the floater unit motions are computed separately in a first step and then imposed as prescribed displacements at the top of the riser finite element model. Subsequently, in the local analysis, the global responses (axial tension and bending moments) are used to compute the stress time series in some points of the riser cross sections. The procedure is illustrated in Figure 1. For a metallic riser, the normal stress time series $\sigma(\mathrm{t})$ in any point of a cross section can be determined by the following expression [10]:

$$
\sigma(\mathrm{t})=\frac{\mathrm{T}(\mathrm{t})}{\mathrm{A}}+\frac{\mathrm{My}(\mathrm{t}) \mathrm{r} \sin (\theta)}{\mathrm{I}_{\mathrm{y}}}+\frac{\mathrm{Mz}(\mathrm{t}) \mathrm{r} \cos (\theta)}{\mathrm{I}_{\mathrm{z}}}
$$

where $\mathrm{T}, \mathrm{My}$, and $\mathrm{Mz}$ are the axial tension and the bending moments, respectively, A and I are the cross sectional area and inertia moments, and $\mathrm{r}$ and $\theta$ are the radius and the 


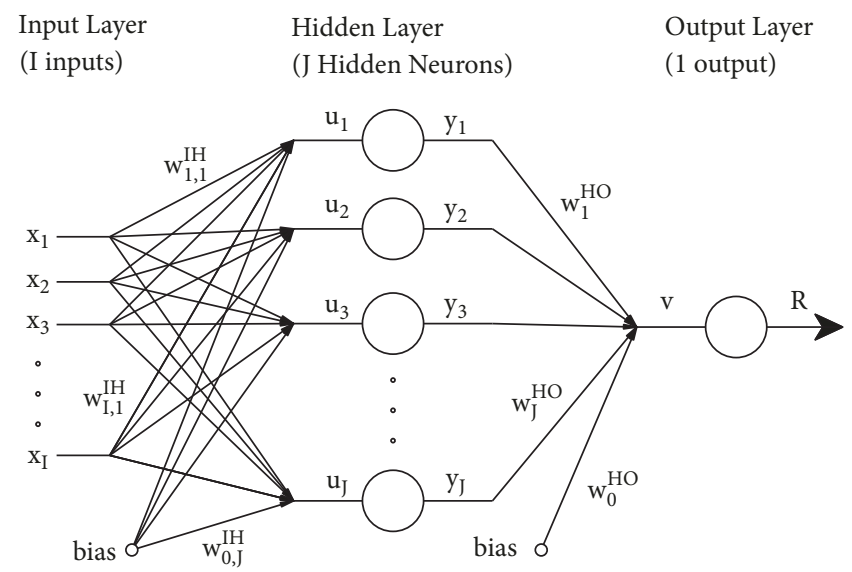

FIGURE 2: ANN architecture.

angle that determines the point where the stress is being computed on the cross section. For fatigue assessment, it is recommended that at least eight points in the internal and external circumferences should be analyzed [10].

After obtaining stresses time series, a cycle counting procedure, such as the Rainflow technique [11], is applied to identify and count the stress cycles for the fatigue damage assessment. The fatigue damage at any point of a cross section is evaluated by means of S-N curves and the Miner-Palmgren damage accumulation rule [12]. Through this procedure, the annual fatigue damage is computed as

$$
\mathrm{D}_{1-\mathrm{yr}}=2920 \sum_{\mathrm{j}=1}^{\mathrm{N}_{\mathrm{ST}}} \sum_{\mathrm{i}=1}^{\mathrm{N}_{\mathrm{C}}{ }^{j}} \frac{\left(\mathrm{S}_{\mathrm{i}}^{\mathrm{j}}\right)^{\mathrm{m}}}{\mathrm{K}} \cdot \gamma_{\mathrm{j}}
$$

where 2920 is the total number of 3 -h sea states in one year, $\mathrm{N}_{\mathrm{C}}{ }^{\mathrm{j}}$ is the number of stress cycles identified by the Rainflow algorithm in the $\mathrm{j}^{\text {th }} 3$ h-loading case (sea state), $\mathrm{N}_{\mathrm{ST}}$ is the total number of loading cases (sea states) considered in the fatigue analyses, $S_{i}{ }^{j}$ is the $i^{\text {th }}$ stress range of the ${ }^{\text {th }}$ loading case, $m$ and $\mathrm{K}$ are the S-N curve parameters, and, finally, $\gamma_{\mathrm{j}}$ is the annual relative frequency of occurrence of the respective loading case $\left(\sum_{j} \gamma_{j}=1\right)$. Commonly, the number of loading cases is very large due to the wave scatter diagrams discretization (mainly for the waves periods) and when all wave directions are considered. The fatigue life (in years) is simply the inverse of the annual fatigue damage.

As mentioned before, the large amount of loading cases combined with the need of long response time series for statistical consistency in the identified stress cycles makes the global analysis the most time-consuming phase of riser fatigue assessment. By using ANNs to predict the global riser response, the procedure presented in this work aims to reduce the computational time associated with this specific phase of the metallic riser fatigue verification.

\section{Artificial Neural Networks}

Artificial Neural Networks (ANNs) constitute a form of mathematical processing of information with architecture and operation similar to the biological neural networks. The main feature of the ANNs is the learning ability, which in practical terms results in applications such as pattern recognition, clustering, classification, optimization, and function approximation, among others [13]. The use of ANNs has been increasing lately to help solving many engineering problems; for instance, see [14-16].

In this work, ANNs are employed to define a mathematical function relating the floating displacements imposed at the riser top with its global responses: axial tension $\mathrm{T}$ and bending moments My and Mz. For such application, the ANN must be trained, using the data and results of a very short simulation provided by the FEA. Then the ANN is used to predict longer loading responses time series.

3.1. ANN Architecture. The most usual ANN architecture applied to function approximation uses three layers: input layer, hidden layer, and output layer. The configuration is outlined on Figure 2. The first layer reads the inputs values of the network. The output layer delivers the responses of the network. The hidden and output layers are composed of neurons (numerical functions). Each connection between elements in neighboring layers contains a weight (synapse weighting). Linked to the hidden and output layers there are also bias parameters, with unitary values.

A neuron $j$ in the hidden layer, for instance, receives an input $u_{j}$, given by

$$
\mathrm{u}_{\mathrm{j}}=\mathrm{w}_{0, \mathrm{j}}^{\mathrm{IH}} \cdot 1+\sum_{\mathrm{i}=1}^{\mathrm{I}}\left(\mathrm{w}_{\mathrm{i}, \mathrm{j}}^{\mathrm{IH}} \cdot \mathrm{x}_{\mathrm{i}}\right)
$$

where $I$ is the number of network inputs, $w_{0, j}^{\mathrm{IH}}$ is the bias weight of the input layer, $\mathrm{w}_{\mathrm{i}, \mathrm{j}}^{\mathrm{IH}}$ is the weight between the input $i$ and the respective neuron $j$ in the hidden layer $(j=1 \ldots J$, $J$ is the total number of hidden layer neurons), and $x_{i}$ is the $i$ network input. Each neuron returns an output $y_{j}$ denoted by

$$
\mathrm{y}_{\mathrm{j}}=\varphi_{\mathrm{H}}\left(\mathrm{u}_{\mathrm{j}}\right)=\varphi_{\mathrm{H}}\left(\mathrm{w}_{0, \mathrm{j}}^{\mathrm{IH}} \cdot 1+\sum_{\mathrm{i}=1}^{\mathrm{I}}\left(\mathrm{w}_{\mathrm{i}, \mathrm{j}}^{\mathrm{IH}} \cdot \mathrm{x}_{\mathrm{i}}\right)\right)
$$




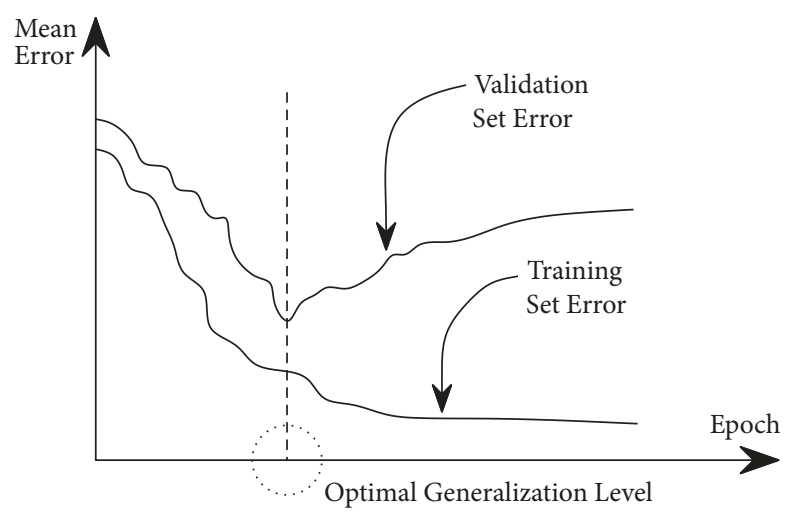

FIGURE 3: Usual behavior of the training and validation errors.

where $\varphi_{\mathrm{H}}($.$) is the so-called activation function, usually$ sigmoid functions like hyperbolic tangent and the logistic functions. For an $\mathrm{ANN}$ with a single output $\mathrm{R}$, this parameter is determined by

$$
\mathrm{R}=\varphi_{\mathrm{O}}(\mathrm{v})=\varphi_{\mathrm{O}}\left(\mathrm{w}_{0}^{\mathrm{HO}} \cdot 1+\sum_{\mathrm{j}=1}^{\mathrm{J}}\left(\mathrm{w}_{\mathrm{j}}^{\mathrm{HO}} \cdot \mathrm{y}_{\mathrm{j}}\right)\right)
$$

where $\varphi_{\mathrm{O}}($.$) is the activation function of the neuron in$ the output layer, usually a linear function. The remaining parameters are defined similarly to those in the hidden layer (see (3)).

3.2. Training. Considering the ANN previously presented and considering the inputs and outputs as time series, the relation between the inputs $x_{i}$ and the output $R$ can be written as

$$
\mathrm{R}(\mathbf{W}, \mathrm{t})=\mathrm{f}(\mathbf{W}, \mathbf{x}(\mathrm{t}))
$$

where $\mathbf{x}(\mathrm{t})$ is a vector containing the time series of the inputs and $\mathbf{W}$ is a matrix containing all ANN connecting weights. Given a sample set of inputs (time series) and the corresponding outputs, the network weights $\mathbf{W}$ can be obtained in such a way that the network output is in agreement to the given outputs sample. This weights adjustment is made by means of an optimization method and is called neural network training. Commonly the optimization problem is expressed by the minimization of the mean square error E, defined as

$$
\begin{aligned}
E(\mathbf{W}) & =\frac{1}{N} \sum_{t=1}^{N}(R(W, t)-v(t))^{2} \\
& =\frac{1}{N} \sum_{t=1}^{N}(f(W, x(t))-v(t))^{2}
\end{aligned}
$$

where $\mathrm{N}$ is the length (number of data points) of the training sample time series and $\mathrm{v}(\mathrm{t})$ is the desired output sample. An iterative optimization procedure, such as the steepest descend method [13], can be employed to obtain optimum values for the weights $\mathbf{W}$. Usually the weights are initialized using small random values. Each iteration in the optimization procedure is called epoch, and the input and output time-series are normalized to facilitate the training procedure $[17,18]$.

Generally, two types of errors can occur during the network training. One is related to the early training stopping, far from the optimum set of weights and originating high prediction errors. The other happens when the ANN is overtrained and it adapts too much to the training set (overfitting). Both situations lead to high prediction errors for new inputs (nontrained data) $[13,17,18]$. Thus, there is an optimum level of training to be achieved. The procedure applied to avoid these errors is to separate part of the training set for the training itself (weights update) and the other part for validation, with separated error functions. Commonly the network error follows the patterns exhibited in Figure 3. The training process must be stopped when the error for the validation set begins to increase [17]. This detail avoids the overfitting and ensures a good network generalization for new inputs.

\section{ANN Model for Global Riser Analysis Responses}

The goal of this work is to apply ANNs for predicting the global riser responses, using an ANN as a surrogate model for the computationally expensive FEA. For a given element of the riser finite element mesh, ANNs are trained to predict the axial tension $\mathrm{T}$ and bending moments $\mathrm{My}$ and $\mathrm{Mz}$ independently:

$$
\begin{aligned}
T(t) & =f_{T}\left(\mathbf{W}_{T}, x(t)\right) \\
M y(t) & =f_{M y}\left(\mathbf{W}_{M y}, x(t)\right) \\
M z(t) & =f_{M z}\left(\mathbf{W}_{M z}, x(t)\right)
\end{aligned}
$$

where $\mathbf{W}_{\mathrm{T}}, \mathbf{W}_{\mathrm{My}}$, and $\mathbf{W}_{\mathrm{Mz}}$ are the optimal weights achieved during the ANN training phase for the three responses and $\mathbf{x}(\mathrm{t})$ are the ANN inputs, i.e., the prescribed translational and rotational floater motions at the riser top. Given the dynamic nature of the analysis, the memory effect of the system (riser) must be taken into account in the ANN model. This aspect can be accomplished in the model by 


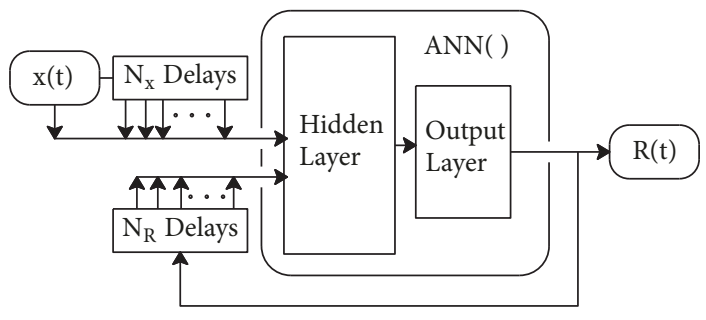

FIGURE 4: ANN-NARX model.

different approaches, e.g., AutoRegressive model (AR) and the EXogenous inputs model (EX). The model employed in this work is a Nonlinear AutoRegressive with eXogenous inputs (NARX) neural network [2]. Then, for the present work, the inputs of a ANN-NARX model consist of the top motions imposed by the floater in the considered time instant, the past values of these displacements, and also the corresponding past values of the respective global response (output). Considering that the time series are discretized at equal time steps $\Delta t$, then the ANN-NARX model is defined as

$$
R(t)=f_{R}\left[\begin{array}{c}
\left\{x(t), x(t-\Delta t), \ldots, x\left(t-N_{x} \Delta t\right)\right\}, \\
\left\{y(t), y(t-\Delta t), \ldots y\left(t-N_{y} \Delta t\right)\right\}, \\
\left\{z(t), z(t-\Delta t), \ldots, z\left(t-N_{z} \Delta t\right)\right\}, \\
\left\{r x(t), r x(t-\Delta t), \ldots, r x\left(t-N_{r x} \Delta t\right)\right\}, \\
\left\{r y(t), r y(t-\Delta t), \ldots, r y\left(t-N_{r y} \Delta t\right)\right\}, \\
\left\{r z(t), r z(t-\Delta t), \ldots, r z\left(t-N_{r z} \Delta t\right)\right\}, \\
\left\{R(t-\Delta t), \ldots R\left(t-N_{R} \Delta t\right)\right\}
\end{array}\right]
$$

where $\mathrm{R}(\mathrm{t})$ represents either $\mathrm{T}(\mathrm{t}), \mathrm{My}(\mathrm{t})$, or $\mathrm{Mz}(\mathrm{t}) ; \mathrm{x}(\mathrm{t}), \mathrm{y}(\mathrm{t})$, and $\mathrm{z}(\mathrm{t})$ represent the global translational motions imposed by the floater; $r x(t), r y(t)$, and $r z(t)$ represent the rotational displacements; $\mathrm{N}_{\mathrm{x}}, \mathrm{N}_{\mathrm{y}}, \mathrm{N}_{\mathrm{z}}, \mathrm{N}_{\mathrm{rx}}, \mathrm{N}_{\mathrm{ry}}$, and $\mathrm{N}_{\mathrm{rz}}$ are the number of time steps delays for the imposed displacements and $N_{R}$ is the number of time steps delays of the response $\mathrm{R}(\mathrm{t})$. In Figure 4 an ANN implemented with the NARX model is outlined.

The ANNs developed in this work were trained and applied using the Neural Networks Toolbox ${ }^{\mathrm{TM}}$ in MATLAB ${ }^{\mathrm{TM}}$ [19]. A hyperbolic tangent function was used as activation function for the neurons in the hidden layer, a linear function was used for the neuron in the output layer, the time series were normalized to have zero mean and unitary standard deviation, the weights were initiated randomly, and the training algorithm utilized was the Levenberg-Marquardt [18, 19].

\section{Hybrid ANN-FEA Methodology}

The main goal of the hybrid ANN-FEA methodology is to be able to conciliate the dynamic model complexity with a reduction of its computational cost. The methodology is named hybrid since a FEA short simulation is used to determine the complex relation between the nonlinear riser response and the top end motions (ANN training). The hybrid ANN-FEA procedure is schematically presented in Figure 5.

Once the floater prescribed motions at the riser top are easily determined by means of the floater RAOs (Response Amplitude Operators), a short length FEA simulation is performed in order to obtain the riser global dynamic response time series. Then, these time series of inputs (top displacements) and outputs (riser responses) are used as training and validation sets for the ANN. After training, the ANN is used to predict the remaining global response, based on the complete time series of the top prescribed motions. In this way, the ANN replaces the onerous FEA numerical integration for a large part of the simulation length.

As usual, in the everyday riser design practice, the lowfrequency floater motions were not considered in the present work (only static offsets were taken into account). However, if it is desired to include low-frequency motions in the analysis, the proposed hybrid FEM-ANN methodology can still be used. In this case, it is only necessary to provide time series of motions and responses used for training and verification, and time series of motions used for prediction, which include the low and wave frequency components. These motions can be obtained, for instance, from a simplified 3D floater motion dynamic analysis (see Guarize [1]).

In this work, the hybrid methodology is applied individually for some elements located at the top and TDZ regions of a SCR, which are the most critical points for fatigue. Individual ANNs are trained and used for each element, for each response parameter considered $(\mathrm{T}(\mathrm{t}), \mathrm{My}(\mathrm{t})$, and $\mathrm{Mz}(\mathrm{t}))$ and for each loading case. This choice of a specific ANN for each loading case takes into consideration that there is a different "catenary" configuration for each load condition and, as a consequence, the TDZ position can change substantially. In general, SCRs present nonlinear and remarkably high bending moments in the touchdown zone (TDZ), but the TDZ is not the same for all loading cases. For instance, a segment that belongs to the TDZ in a "far" loading case may not be affected by bending in a "near" loading case. Then, considering many loading conditions, the stress response for a given riser point in the touchdown zone is not well behaved as it almost is for the suspended riser segment. Therefore, an ANN trained for predicting the riser stresses at the TDZ, employing the combination of different signals from different sea state conditions, may not lead to good results for sea states not present in the training process. 

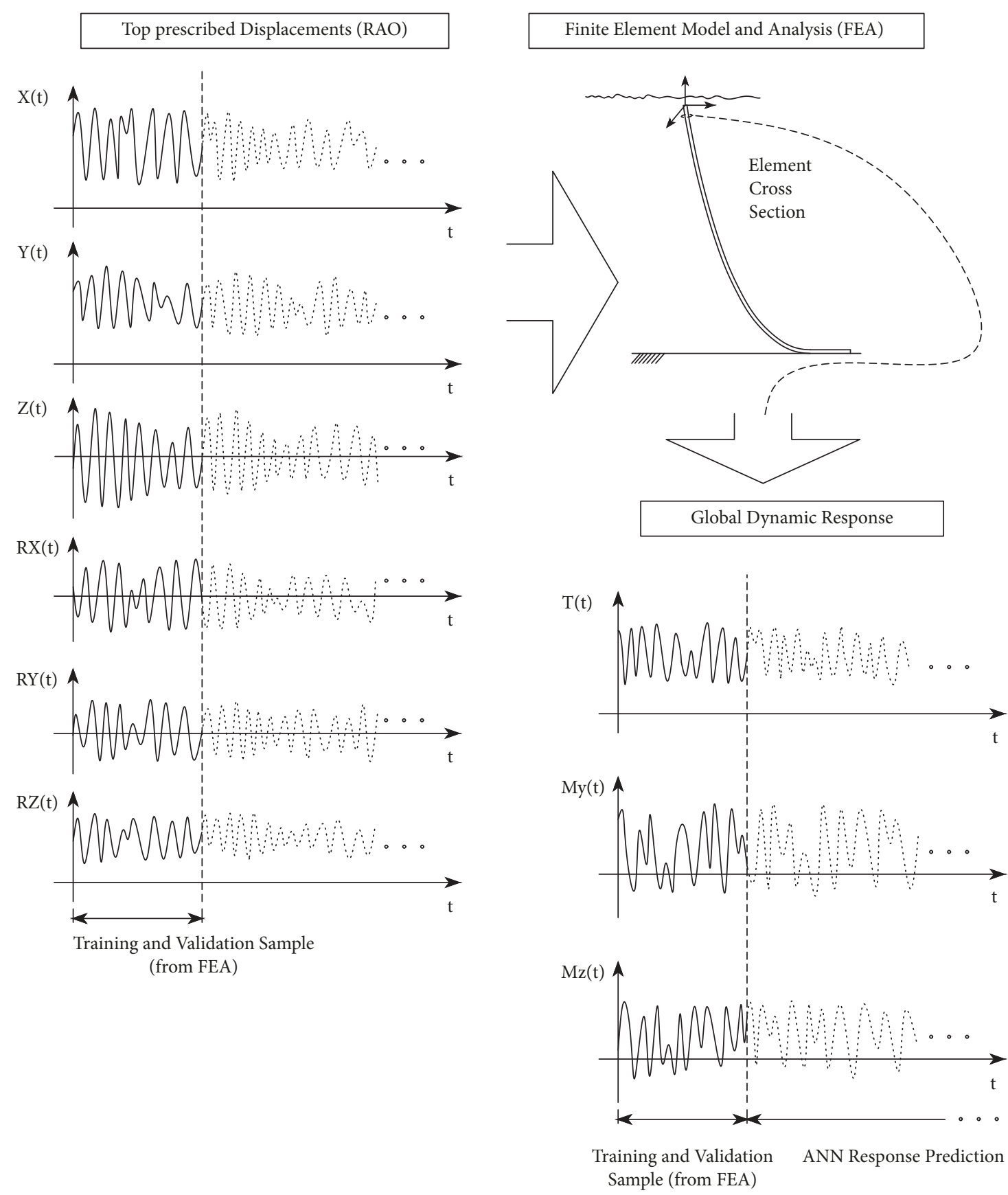

FIgURE 5: Hybrid ANN-FEA method.

\section{Case Study}

The hybrid ANN-FEA procedure is applied to compute the wave-induced fatigue life of some elements at the top and at the TDZ region of a steel catenary riser (SCR) (see Figure 6). This metallic riser is described by Senra [20] and consists of a $10.7^{\prime \prime}$ gas export SCR connected to a semisubmersible platform in a water depth of $910 \mathrm{~m}$. The SCR model has a flexjoint in the connection. The physical and geometrical properties of the model are found in Table 1, while the information about the finite element mesh employed is presented in Table 2. The semisubmersible platform heading is $20^{\circ}$ (in relation to true north) and the riser azimuth is $192.11^{\circ}$ (see Figure 6).

The loading cases selected to perform the fatigue analysis are defined by static offsets and current and wave loadings. The same current profile was adopted for all loading cases considered in the fatigue analysis. This profile is triangular with a superficial velocity of $0.7 \mathrm{~m} / \mathrm{s}$, propagating to the south direction. The offset magnitude is a function of the significant wave height $\mathrm{Hs}$ of the respective load case, and it is proportional to $2.5 \%$ of the SWL (Still Water Level) for the 

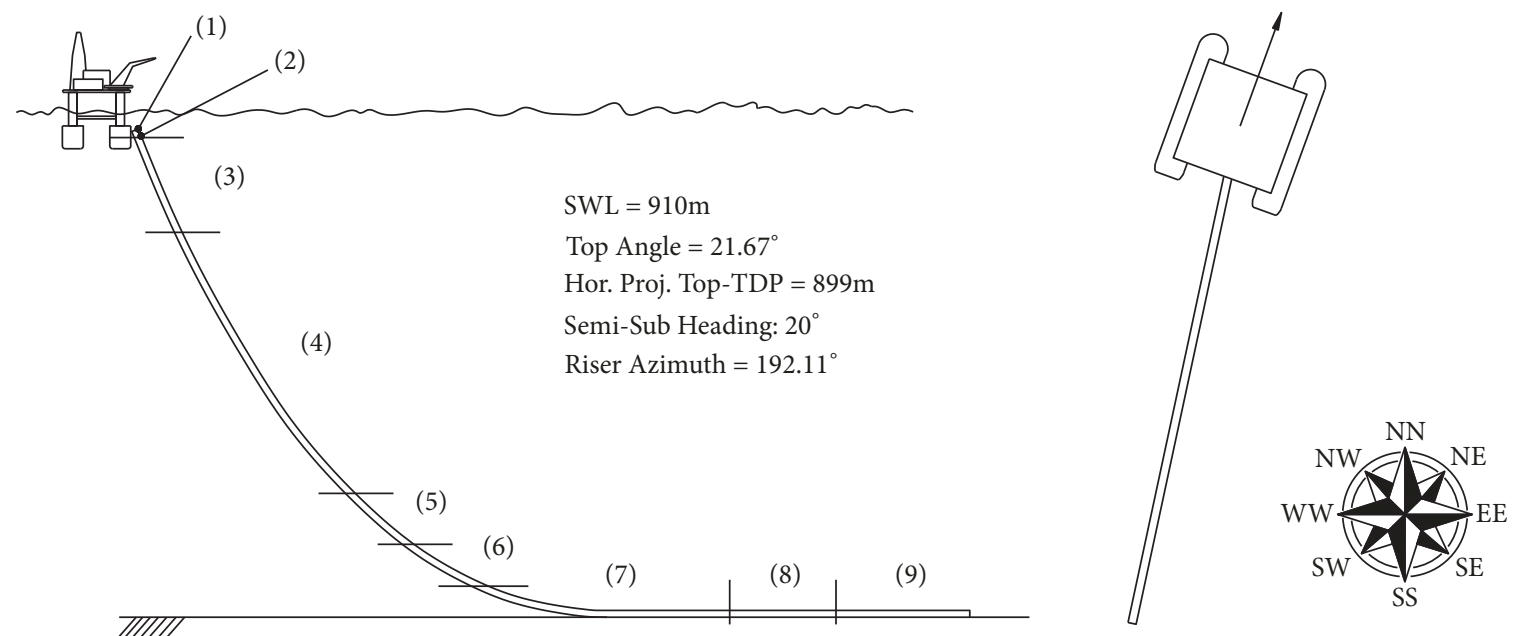

FIGURE 6: SCR model used in the study case.

TABLE 1: SCR properties.

\begin{tabular}{lc}
\hline Parameter & Value \\
\hline External Diameter $(\mathrm{m})$ & $0.273\left(10.7^{\prime \prime}\right)$ \\
Internal Diameter $(\mathrm{m})$ & $0.232\left(9.13^{\prime \prime}\right)$ \\
Elasticity Module $\left(\mathrm{kN} / \mathrm{m}^{2}\right)$ & $2.07 \mathrm{E}+08$ \\
Specific Weight $\left(\mathrm{kN} / \mathrm{m}^{3}\right)$ & 77.00 \\
Internal Fluid Weight $\left(\mathrm{kN} / \mathrm{m}^{3}\right)$ & 2.00 (gas) \\
Top Internal Pressure $(\mathrm{MPa})$ & 18.00 \\
Soil vertical stiffness $(\mathrm{kN} / \mathrm{m})$ & 1000.00 \\
Riser Top Angle & $21.67^{\circ}$ \\
Riser Azimuth & $192.11^{\circ}$ \\
\hline
\end{tabular}

highest significant wave height of the wave scatter diagram. The offset direction is coincident with the wave propagation direction. The waves selected are divided into 8 incidence directions, with 11 waves (sea states) for each direction. The frequency of occurrence for each incidence direction is presented in Table 3, while the irregular wave parameters and their frequency of occurrence for each direction are found in Table 4. The JONSWAP spectrum was adopted to represent the sea surface elevation. Then, the fatigue analysis requires the irregular wave simulations for 88 loading cases. In fact, this is a simplified representation of wave data which was employed in order to make all comparisons performed in this work possible. Once the current is the same and the offset is defined by the significant wave height, the loading case is identified herein by the wave name. Specifically, a loading case identified as NE03 is a case where the wave has a northeast incidence direction, a significant wave height Hs of $1.25 \mathrm{~m}$, a spectral peak period Tp of $7.02 \mathrm{~s}$, and an annual frequency of occurrence of $2.17 \%$ (Tables 3 and 4 ).

A usual FEA-based fatigue analysis was initially performed for comparison purposes and it was also used to identify the elements with the lower fatigue life at the top and TDZ regions, ensuring that the hybrid methodology is applied (and evaluated) to the critical structure points. The fatigue assessment of the entire structure was based on the DNV E S-N curve [21].

6.1. ANN Architecture and Training. As it can be observed from the definition of an ANN, the architecture of the network is defined by the number of neurons in the hidden layer and the time-series steps delays. As said before, a specific ANN is established for each response parameter, for each loading case, and for each element along the structure. In this work an iterative process, described below, was employed to define the architecture of each ANN.

It is assumed that the number of delays is the same for all input and output time series, so the two open parameters to define the ANN architecture are the number of step delays and the number of neurons in the hidden layer. Based on initial tests and previous works [1-3, 5], a sequential trial set of numbers for these two parameters is specified. The sets employed were as follows (hidden neurons/delays): 10/10, $10 / 5,10 / 2,12 / 10,12 / 5,12 / 2,15 / 10,15 / 5,15 / 2,20 / 10,20 / 5$, $20 / 2,5 / 10,5 / 5,5 / 2,10 / 1,12 / 1,20 / 1,3 / 10,3 / 5,3 / 2,5 / 30,5 / 40$, and $5 / 50$. The effectiveness of a given architecture (for each response loading case in a structure point) is assessed by comparing the ANN predicted response (trained with one of the trial sets) with the "true" FEA-based response used for training the network.

The assessment is performed based on statistical results, as described in Algorithm 1. If the trained ANN results satisfy the conditions specified in Algorithm 1, the ANN is taken as the surrogate model for the hybrid method. If not, other attempts are made using the same trial architecture, given the random weights initialization $[17,18]$. If the maximum attempts per trial set are reached without complying with the conditions, the following trial set is evaluated, as outlined in Algorithm 1. The acceptable tolerances given in Algorithm 1 were determined by means of initial trial and error tests.

The time series were discretized in steps of $0.25 \mathrm{~s}$ and the FEM-based simulation (used for training/validation) was 
TABLE 2: SCR finite element mesh.

\begin{tabular}{lcccc}
\hline Seg & Length $(\mathbf{m})$ & Number of elements & Element initial length $(\mathbf{m})$ & Element final length $(\mathbf{m})$ \\
\hline 1 & 1.102 & 14 & 0.08 & 0.08 \\
2 & 10 & 112 & 0.08 & 0.10 \\
3 & 200 & 364 & 0.10 & 1.00 \\
4 & 600 & 600 & 1.00 & 1.00 \\
5 & 150 & 171 & 1.00 & 0.75 \\
6 & 150 & 240 & 0.75 & 0.50 \\
7 & 500 & 1000 & 0.50 & 0.50 \\
8 & 200 & 160 & 0.50 & 2.00 \\
9 & 255.847 & 73 & 2.00 & 5.00 \\
\hline
\end{tabular}

For each loading case response do

nset $=1$

While [nt $<=$ MaxTries and nset $<=$ QttSet] do

Train ANN

Apply ANN

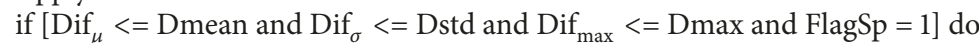

TestFlag $=1$

else do

TestFlag $=0$

end

if $[$ TestFlag $=1]$ do

nt $=1000$, nset $=1000$ (successful training!)

elseif [TestFlag $=0$ and $n t<$ MaxTries and nset $<=$ QttSet $]$ do

$\mathrm{nt}=\mathrm{nt}+1$

elseif $[$ TesteFlag $=0$ and $n t=$ MaxTries and nset $<$ QttSet $]$ do

$\mathrm{nt}=1$

nset $=$ nset +1

elseif $[$ TesteFlag $=0$ and $n t=$ MaxTries and nset $=$ QttSet $]$ do

end

$\mathrm{nt}=1000$, nset $=1000$ (unsuccessful training!)

end

$\mathrm{nt}=$ current number of attempt per trial set

nset $=$ current trial set number

MaxTries = maximum number of attempt per trial set (5)

QttSet = trial sets quantity (24)

Dmean $=$ acceptable difference for mean $(5 \%)$

Dstd $=$ acceptable difference for standard deviation ( $8 \%$ for axial tension, $6 \%$ for moments)

Dmax $=$ acceptable difference for maximum observed value (300\% for axial tension, $200 \%$ for moments)

FlagSp = Energy spectrum flag, equal to 1 if no significant energy is observed in high frequencies, for ANN response

$\operatorname{Dif}_{\mu}=100 \% \cdot \frac{\left|\mu_{\text {training }}-\mu_{\text {ANN }}\right|}{\mu_{\text {training }}}$

$\operatorname{Dif}_{\sigma}=100 \% \cdot \frac{\left|\sigma_{\text {training }}-\sigma_{\text {ANN }}\right|}{\sigma_{\text {training }}}$

$\mathrm{Dif}_{\max }=100 \% \cdot \frac{\left|\mathrm{Max}_{\text {training }}-\mathrm{Max}_{\mathrm{ANN}}\right|}{\mathrm{Max}_{\text {training }}}$

$\mu_{\text {training }}, \mu_{\mathrm{ANN}}=$ mean values of the training and predicted time series

$\sigma_{\text {training }}, \sigma_{\mathrm{ANN}}=$ standard deviation values of the training and predicted time series

$\operatorname{Max}_{\text {training }}, \operatorname{Max}_{\mathrm{ANN}}=$ observed maximum values of the training and predicted time series

Algorithm 1: ANN training procedure. 


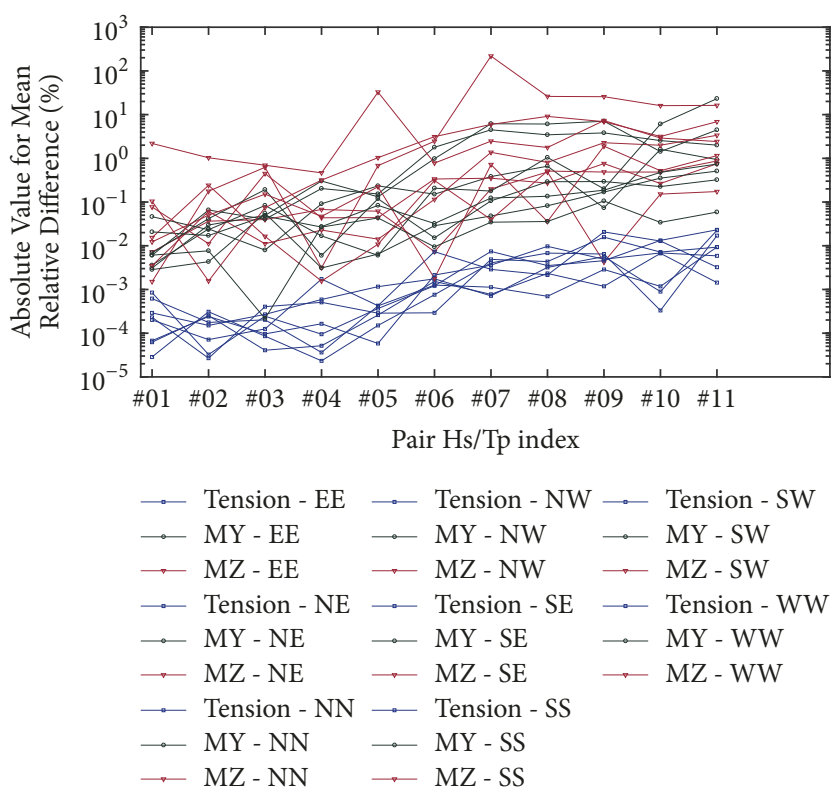

FIGURE 7: Relative differences for the response mean values (critical riser top element).

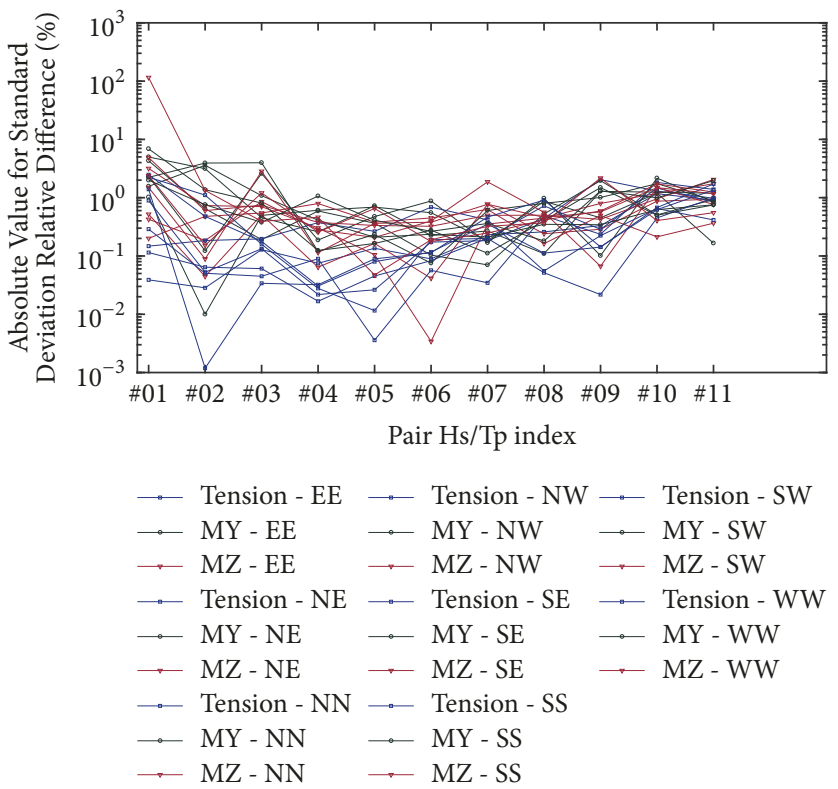

FIGURE 8: Relative differences for the response standard deviation values (critical riser top element).

500s long. The training is done using $80 \%$ of the sample, while the remaining $20 \%$ is used for validation. The training is stopped when the validation error starts to increase.

The time spent in this batch training procedure is quite low, mainly for those points located close to the riser top, where usually the first ANN architecture trial leads to very good results. For some TDZ response cases, the searching for a good configuration results in more verifications and tests of different ANN configurations; however the training time spent is very low when compared to the full-fatigue FEA (generally speaking, no more than 1 minute of CPU time is spent to train an ANN).
6.2. ANN Predicted Responses. With the trained ANNs available, the riser global responses (axial tension and bending moments) were predicted till 10800 seconds (3 hours), and the obtained results were compared with those obtained by the complete FEA, for the elements with the lowest fatigue lives at the top and TDZ regions, the critical ones. The results are compared through the relative difference for any response statistical parameter estimation or fatigue damage by the generic relationship: ((Hybrid ${ }_{\mathrm{ANN}-\mathrm{FEA}}$ ResultFEA Result)/|FEA Result|) $* 100 \%$.

In Figures 7 and 8 the relative differences for the mean $(\mu)$ and standard deviation $(\sigma)$ of the response parameters 


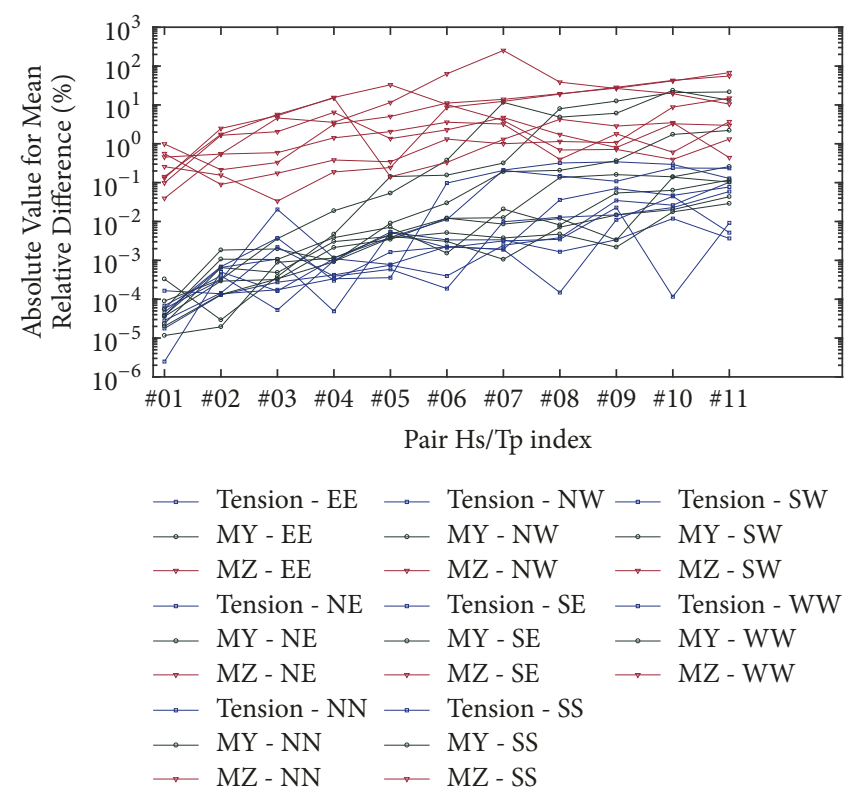

FIgURE 9: Relative differences for the response mean values (critical riser TDZ element).

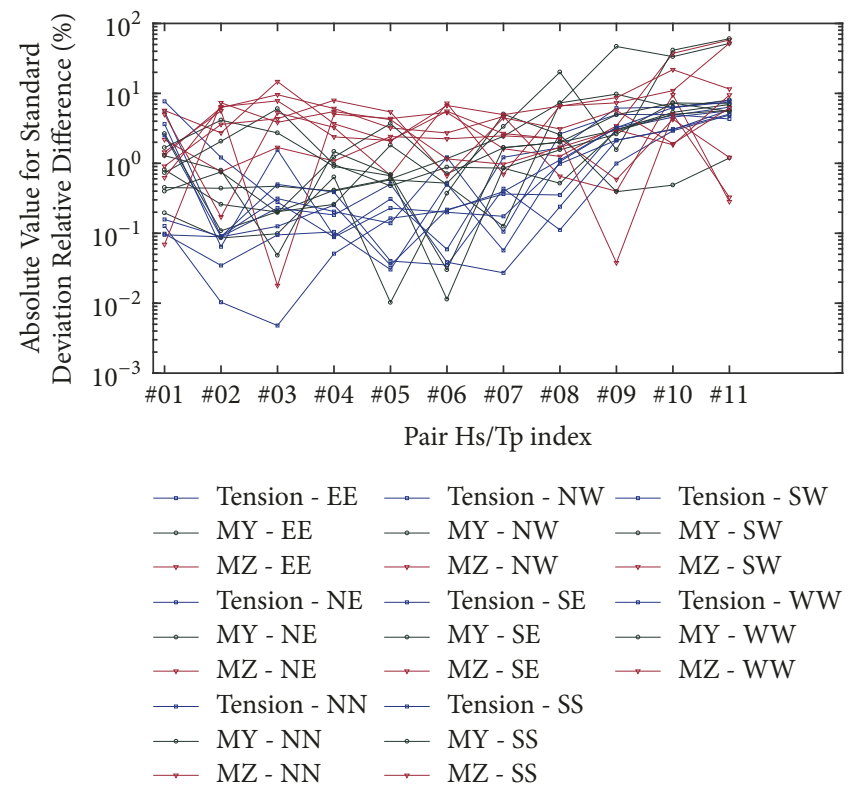

FIGURE 10: Relative differences for the response standard deviation values (critical riser TDZ element).

(absolute values) are presented, respectively, for each loading case, for the critical element cross section at the riser top. Figures 9 and 10 present the same results for the riser critical cross section located at the TDZ. As can be observed from the figures, the relative differences found for the axial tension are, in general, lower than those for the bending moments. The differences are larger for the critical point located at the TDZ than those for the riser top. This is expected since the TDZ is strongly influenced by nonlinearities due to the complex soilstructure interaction. However, for the majority of the cases, the differences can be considered low.
6.3. Fatigue Analysis Results. From the predicted global responses, the stress time series are determined for the selected SCR elements. Figure 11 shows a short window of a stress time series obtained by the hybrid ANN-FEA method and the corresponding one provided by the full FEA for the most critical point on the element with the lowest fatigue life at the top. The stress time series is taken from the loading case that contributes most to the total fatigue damage. Figure 12 shows similar comparison for the riser TDZ critical element cross section. As can be seen, the agreement between the ANN prediction and FEA results is remarkable. 


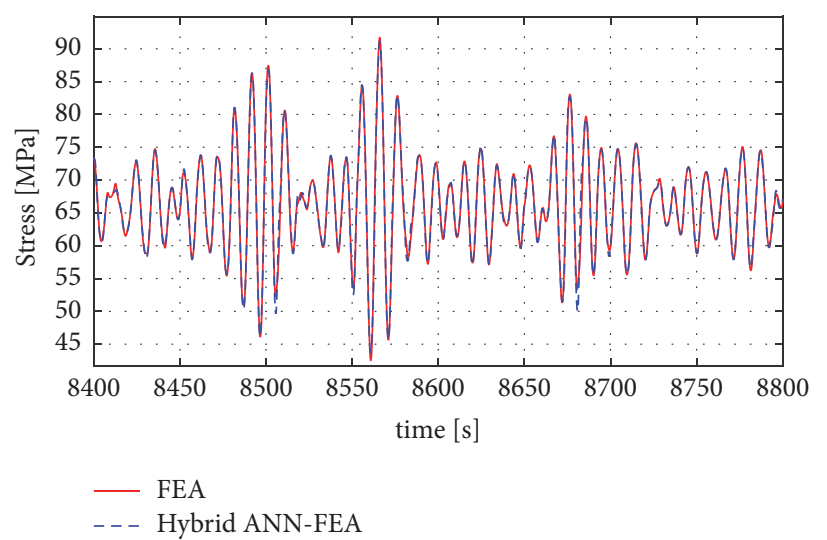

FIgURE 11: Stresses time series, critical point, and loading case in the riser critical element at top.

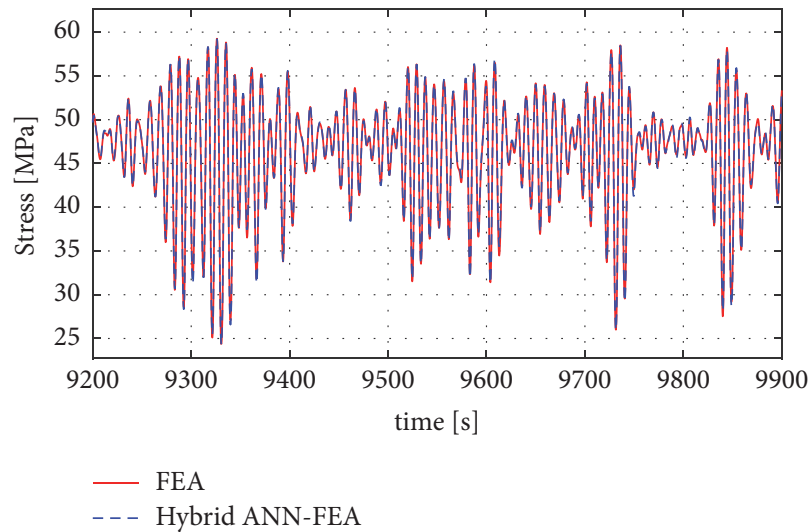

FIGURE 12: Stresses time series, critical point, and loading case in the riser critical element at TDZ.

TABLE 3: Waves incidence directions.

\begin{tabular}{|c|c|c|}
\hline \multicolumn{2}{|c|}{ Incidence Direction } & \multirow{2}{*}{$\frac{\text { Frequency (\%) }}{26.0}$} \\
\hline NN & North & \\
\hline NE & Northeast & 9.0 \\
\hline $\mathrm{EE}$ & East & 1.5 \\
\hline SE & Southeast & 1.5 \\
\hline SS & South & 10.0 \\
\hline SW & Southwest & 23.0 \\
\hline WW & West & 13.0 \\
\hline NW & Northwest & 16.0 \\
\hline \multicolumn{2}{|c|}{ Sum: } & 100.0 \\
\hline
\end{tabular}

Table 5 presents the correlation coefficients between ANN predicted and FEA stress time series for each loading case in the most critical point of the cross section at the riser top. Table 6 presents similar results for the riser critical element at the TDZ. For the riser top, in general, there is a very good correlation between both results. The lower correlation values are observed for loading cases with mild waves. For the TDZ region the results are also good, although some lower correlation values are observed for some scattered loading cases. In these cases, the combination of the top imposed displacements and the offset leads to considerable nonlinear responses for the specific element, especially for the My bending moment.

Figure 13 presents the annual fatigue damage for the loading cases related to waves coming from north direction (the direction with the highest accumulated damage) for the riser top critical element. Figure 14 shows similar results for the critical element located at the TDZ. These two figures show very good agreement between the hybrid methodology and the FEA results for the computed fatigue damage.

Finally, in Figure 15 the fatigue life computed by the hybrid ANN-FEA methodology is plotted together with the results given by the full FEA and also with the relative differences (\%) for all cross sections elements evaluated on the top region. Figure 16 shows similar results for all cross sections located at the TDZ where fatigue results have been assessed. It is observed that the fatigue life differences are lower for the top region, where the maximum difference is $2.2 \%$. For the elements located at the TDZ, higher differences are found (maximum difference is $11.0 \%$ ). However, for the elements near the region with the lowest fatigue life in the TDZ, the maximum difference observed was lower, i.e., $3.2 \%$.

The fatigue damage and life of the critical riser elements identified by both methods are summarized in Table 7 . For the top zone, the critical riser point identified for both 
TABLE 4: Wave parameters.

\begin{tabular}{lcccc}
\hline Index \# & Hs (m) & Tz $(\mathbf{s})$ & Tp (s) & Occurrence Frequency (\%) \\
\hline 01 & 0.25 & 3.75 & 4.95 & $1.74 \mathrm{E}-03$ \\
02 & 0.75 & 4.75 & 6.33 & $3.40 \mathrm{E}+00$ \\
03 & 1.25 & 5.25 & 7.02 & $2.41 \mathrm{E}+01$ \\
04 & 1.75 & 6.25 & 8.41 & $3.33 \mathrm{E}+01$ \\
05 & 2.25 & 6.75 & 9.11 & $2.21 \mathrm{E}+01$ \\
06 & 2.75 & 7.25 & 9.81 & $1.05 \mathrm{E}+01$ \\
07 & 3.25 & 7.75 & 10.51 & $4.20 \mathrm{E}+00$ \\
08 & 3.75 & 8.25 & 11.21 & $1.60 \mathrm{E}+00$ \\
09 & 4.25 & 8.75 & 11.91 & $5.63 \mathrm{E}-01$ \\
10 & 4.75 & 9.25 & 12.62 & $2.01 \mathrm{E}-01$ \\
11 & 5.25 & 9.75 & 13.32 & $3.40 \mathrm{E}-02$ \\
\hline
\end{tabular}

TABLE 5: Stress correlation coefficients (riser top cross section).

\begin{tabular}{|c|c|c|c|c|c|c|}
\hline$\#$ & $\begin{array}{l}\text { Index } \\
\text { Hs }(\mathbf{m})\end{array}$ & $\mathrm{Tp}(\mathrm{s})$ & $\mathrm{EE}$ & NE & NN & NW \\
\hline 01 & 0.25 & 4.95 & 0.9952 & 0.9332 & 0.8018 & 0.9759 \\
\hline 02 & 0.75 & 6.33 & 0.9994 & 0.9787 & 0.9631 & 0.9977 \\
\hline 03 & 1.25 & 7.02 & 0.9995 & 0.9846 & 0.9845 & 0.9833 \\
\hline 04 & 1.75 & 8.41 & 0.9992 & 0.9977 & 0.9975 & 0.9984 \\
\hline 05 & 2.25 & 9.11 & 0.9993 & 0.9982 & 0.9954 & 0.9986 \\
\hline 06 & 2.75 & 9.81 & 0.9992 & 0.9985 & 0.9976 & 0.9990 \\
\hline 07 & 3.25 & 10.51 & 0.9975 & 0.9990 & 0.9982 & 0.9992 \\
\hline 08 & 3.75 & 11.21 & 0.9985 & 0.9986 & 0.9988 & 0.9979 \\
\hline 09 & 4.25 & 11.91 & 0.9989 & 0.9965 & 0.9983 & 0.9992 \\
\hline 10 & 4.75 & 12.62 & 0.9981 & 0.9984 & 0.9987 & 0.9978 \\
\hline 11 & 5.25 & 13.32 & 0.9978 & 0.9987 & 0.9976 & 0.9986 \\
\hline \multicolumn{3}{|c|}{ Index } & SE & SS & SW & WW \\
\hline 01 & 0.25 & 4.95 & 0.9613 & 0.7846 & 0.7549 & 0.9841 \\
\hline 02 & 0.75 & 6.33 & 0.9740 & 0.8935 & 0.9230 & 0.9991 \\
\hline 03 & 1.25 & 7.02 & 0.9791 & 0.9328 & 0.9841 & 0.9992 \\
\hline 04 & 1.75 & 8.41 & 0.9968 & 0.9939 & 0.9977 & 0.9977 \\
\hline 05 & 2.25 & 9.11 & 0.9983 & 0.9964 & 0.9988 & 0.9988 \\
\hline 06 & 2.75 & 9.81 & 0.9992 & 0.9988 & 0.9973 & 0.9989 \\
\hline 07 & 3.25 & 10.51 & 0.9994 & 0.9981 & 0.9992 & 0.9980 \\
\hline 08 & 3.75 & 11.21 & 0.9994 & 0.9988 & 0.9993 & 0.9982 \\
\hline 09 & 4.25 & 11.91 & 0.9993 & 0.9991 & 0.9967 & 0.9976 \\
\hline 10 & 4.75 & 12.62 & 0.9994 & 0.9984 & 0.9993 & 0.9980 \\
\hline 11 & 5.25 & 13.32 & 0.9974 & 0.9989 & 0.9991 & 0.9786 \\
\hline
\end{tabular}

methods is the same, but for the TDZ the hybrid ANN-FEA method identified a point which is 1.0 meter far apart from the critical one identified by FEA. Despite this, in practical terms, the distance is too short (given the SWL and the riser length) and the values estimated are in closer agreement.

\section{Conclusions and Final Remarks}

In this paper the use of a hybrid ANN-FEA methodology was investigated with the aim of speeding up the wave-induced fatigue analysis of Steel Catenary Risers. In general, at least for early design stages, this methodology predicted quite good global responses for elements evaluated on top and TDZ regions of the SCR investigated. Consequently, the estimated fatigue lives for these regions were also predicted with very good accuracy. The higher differences occur in some elements located at the TDZ. However, for the critical element in this region (lowest fatigue life), the predicted results were as good as the ones obtained for the SCR top. 
TABLE 6: Stress correlation coefficients (riser TDZ cross section).

\begin{tabular}{|c|c|c|c|c|c|c|}
\hline \multicolumn{3}{|c|}{ Index } & \multirow{2}{*}{$\mathrm{EE}$} & \multirow{2}{*}{$\mathrm{NE}$} & \multirow{2}{*}{ NN } & \multirow{2}{*}{ NW } \\
\hline$\#$ & Hs (m) & $\mathrm{Tp}(\mathrm{s})$ & & & & \\
\hline 01 & 0.25 & 4.95 & 0.9922 & 0.9489 & 0.9253 & 0.9857 \\
\hline 02 & 0.75 & 6.33 & 0.9985 & 0.9968 & 0.9983 & 0.9972 \\
\hline 03 & 1.25 & 7.02 & 0.9970 & 0.9699 & 0.9986 & 0.9986 \\
\hline 04 & 1.75 & 8.41 & 0.9953 & 0.9992 & 0.9992 & 0.9858 \\
\hline 05 & 2.25 & 9.11 & 0.9971 & 0.9966 & 0.9990 & 0.9971 \\
\hline 06 & 2.75 & 9.81 & 0.9963 & 0.9980 & 0.9979 & 0.9944 \\
\hline 07 & 3.25 & 10.51 & 0.9940 & 0.9970 & 0.9893 & 0.9917 \\
\hline 08 & 3.75 & 11.21 & 0.9744 & 0.9614 & 0.9520 & 0.9909 \\
\hline 09 & 4.25 & 11.91 & 0.9597 & 0.8650 & 0.9805 & 0.9948 \\
\hline 10 & 4.75 & 12.62 & 0.9803 & 0.9356 & 0.9851 & 0.9520 \\
\hline 11 & 5.25 & 13.32 & 0.9776 & 0.9610 & 0.9954 & 0.9728 \\
\hline \# & $\begin{array}{l}\text { Index } \\
\text { Hs (m) }\end{array}$ & $\operatorname{Tp}(s)$ & SE & SS & SW & WW \\
\hline 01 & 0.25 & 4.95 & 0.9906 & 0.9343 & 0.9438 & 0.9897 \\
\hline 02 & 0.75 & 6.33 & 0.9806 & 0.8037 & 0.9570 & 0.9803 \\
\hline 03 & 1.25 & 7.02 & 0.9722 & 0.9613 & 0.7965 & 0.9669 \\
\hline 04 & 1.75 & 8.41 & 0.9742 & 0.9888 & 0.9808 & 0.9615 \\
\hline 05 & 2.25 & 9.11 & 0.9843 & 0.9934 & 0.9892 & 0.9671 \\
\hline 06 & 2.75 & 9.81 & 0.9890 & 0.9956 & 0.9930 & 0.9789 \\
\hline 07 & 3.25 & 10.51 & 0.9907 & 0.9959 & 0.9940 & 0.9827 \\
\hline 08 & 3.75 & 11.21 & 0.9934 & 0.9919 & 0.9952 & 0.9803 \\
\hline 09 & 4.25 & 11.91 & 0.9902 & 0.9937 & 0.9955 & 0.9446 \\
\hline 10 & 4.75 & 12.62 & 0.9458 & 0.9894 & 0.9750 & 0.9457 \\
\hline 11 & 5.25 & 13.32 & 0.9533 & 0.9898 & 0.9729 & 0.9360 \\
\hline
\end{tabular}

TABLE 7: Fatigue damage and life (riser top and TDZ critical points).

\begin{tabular}{lcccc}
\hline Region & Method & Dist. from top $(\mathbf{m})$ & Annual Damage & Fatigue Life (yr) \\
\hline \multirow{2}{*}{ Top } & ANN-FEA & 1.10 & $2.39 \mathrm{E}-02$ & 41.8 \\
& FEA & 1.10 & $2.43 \mathrm{E}-02$ & 41.1 \\
\multirow{2}{*}{ TDZ } & ANN-FEA & 1341.60 & $6.68 \mathrm{E}-03$ & 149.8 \\
& FEA & 1342.60 & $6.63 \mathrm{E}-03$ & 150.9 \\
\hline
\end{tabular}

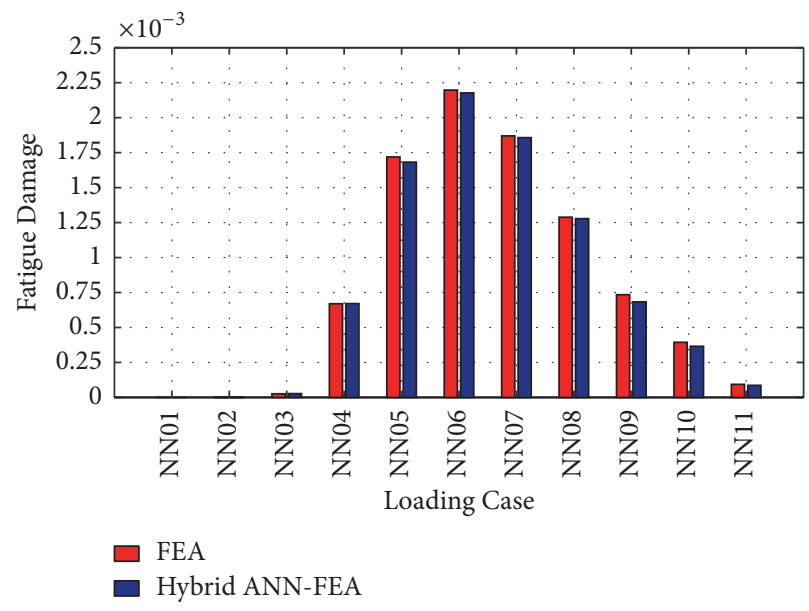

Figure 13: Fatigue damage for north direction waves. Riser top critical element. 


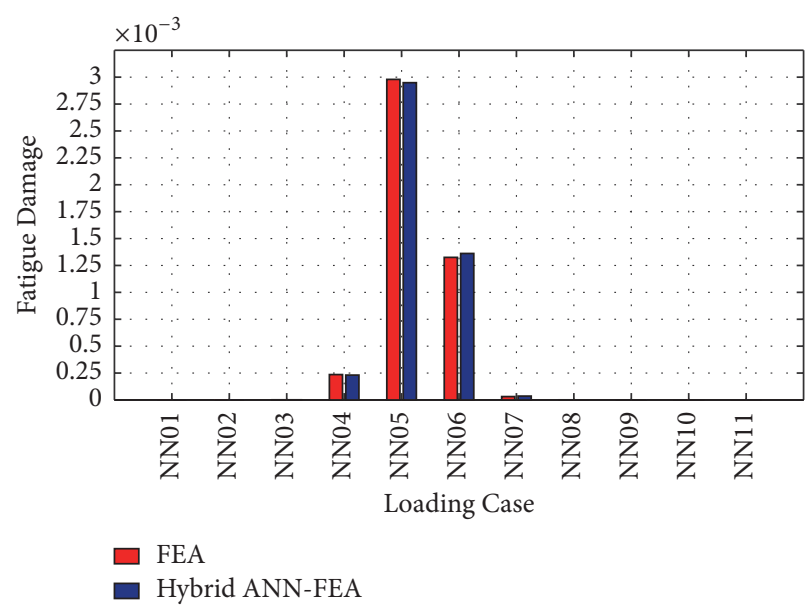

FIGURE 14: Fatigue damage for north direction waves. Riser TDZ critical element.
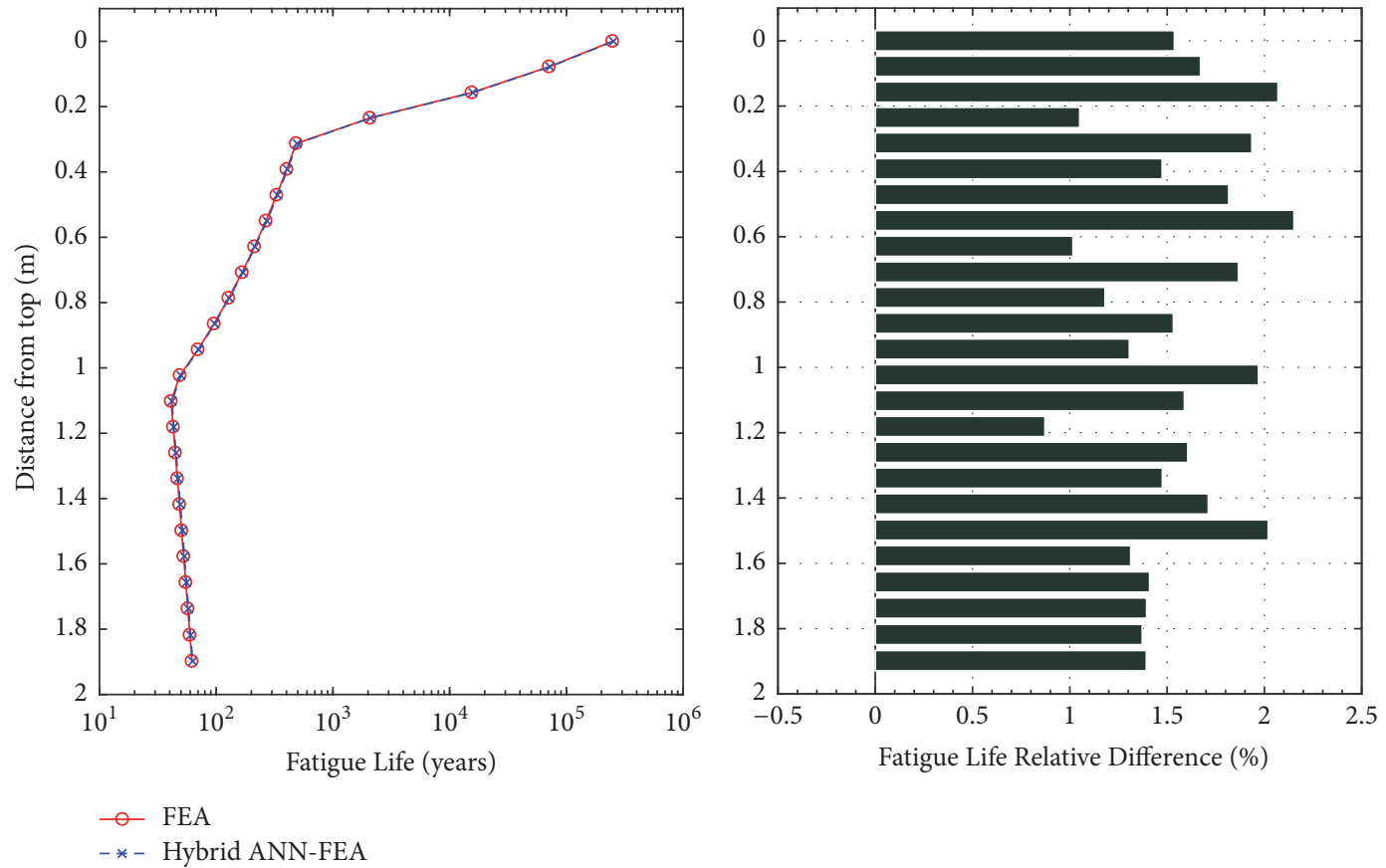

FIGURE 15: Fatigue lives and relative differences for elements at top riser.

In this work, for each riser element, each loading case, and each global response, an ANN was trained using a $500 \mathrm{~s}$ long response time series provided by FEA. Then, the predicted response is generated by the ANN till 10800 seconds using the displacements determined analytically by RAOs. Once the computational time associated with the fatigue damage evaluation and the ANNs training is very low compared to the full global FEA, the hybrid ANN-FEA method can be approximately 20-22 times faster than the usual FEA procedure. The results obtained in this study are very encouraging to go further in this topic, focusing mainly on the development of an automatic procedure to set up the ANN architecture, training, and evaluation, in order to make the practical application of the methodology easier.

\section{Data Availability}

The data used to support the findings of this study are available from the corresponding author upon request.

\section{Conflicts of Interest}

The authors declare that they have no conflicts of interest.

\section{Acknowledgments}

The authors wish to acknowledge the support of Laboratory of Analysis and Reliability of Offshore Structures, Civil 

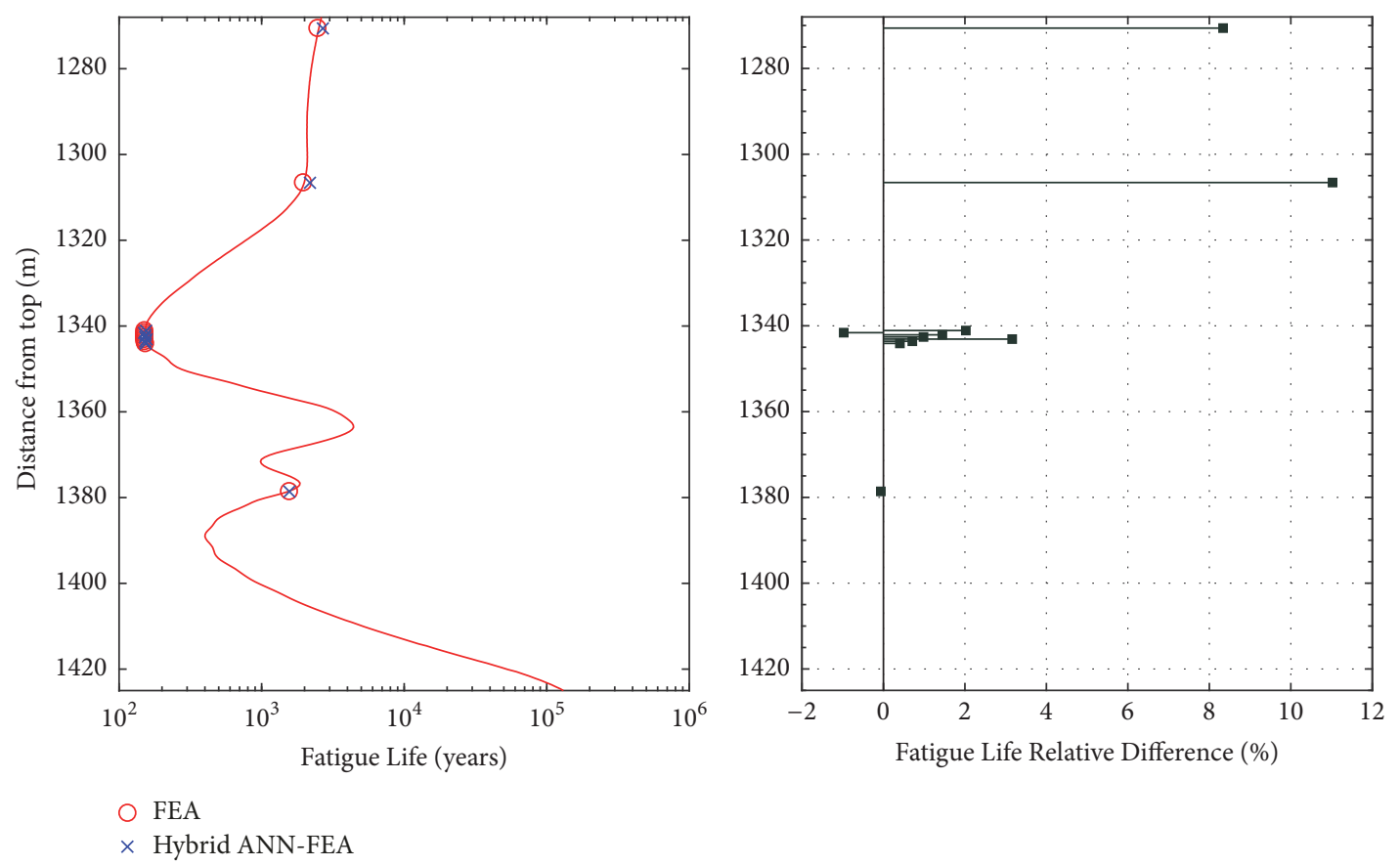

Figure 16: Fatigue lives and relative differences for elements at the TDZ.

Engineering Program, COPPE, Federal University of Rio de Janeiro, for the development of this work.

\section{References}

[1] R. Guarize, N. A. F. Matos, L. V. S. Sagrilo, and E. C. P. Lima, "Neural networks in the dynamic response analysis of slender marine structures," Applied Ocean Research, vol. 29, no. 4, pp. 191-198, 2007.

[2] A. C. de Pina, A. A. de Pina, C. H. Albrecht, B. S. Leite Pires de Lima, and B. P. Jacob, "ANN-based surrogate models for the analysis of mooring lines and risers," Applied Ocean Research, vol. 41, pp. 76-86, 2013.

[3] N. H. Christiansen, P. E. Torbergsen Voie, J. Hogsberg, and N. Sodahl, "Efficient mooring line fatigue analysis using a hybrid method time domain simulation scheme," in Proceedings of the ASME 2013 32nd International Conference on Ocean, Offshore and Arctic Engineering, Nantes, France.

[4] N. H. Christiansen, P. E. Voie, O. Winther, and J. Høgsberg, "Comparison of Neural Network Error Measures for Simulation of Slender Marine Structures," Journal of Applied Mathematics, vol. 2014, pp. 1-11, 2014.

[5] V. Chaves, L. V. Sagrilo, V. R. Silva, and M. A. Vignoles, "Artificial Neural Networks Applied to Flexible Pipes Fatigue Calculations," in Proceedings of the ASME 2015 34th International Conference on Ocean, Offshore and Arctic Engineering, p. V05BT04A022, St. John's, Newfoundland, Canada.

[6] Y. Kim, "Prediction of the dynamic response of a slender marine structure under an irregular ocean wave using the NARX-based quadratic Volterra series," Applied Ocean Research, vol. 49, pp. 42-56, 2015.

[7] Y. Kim, "Finite memory quadratic Volterra model for the response prediction of a slender marine structure under a
Morison load," Journal of Fluids and Structures, vol. 56, pp. 75$88,2015$.

[8] “DNV-OS-F201 (Det Norsk Veritas)," Dynamic Risers, Hovik, Norway, 2010.

[9] API 5L (American Petroleum Insitute), Specification for Line Pipe, Washington, USA, 2000.

[10] DNV-RP-F204 (Det Norsk Veritas), Riser Fatigue, Norway, 2010.

[11] Standard Practices for Cycle Counting in Fatigue Analsysis, ASTM (American Society for Testing and Materials), West Conshohocken, USA, 2011.

[12] A. Almar-Naess, Fatigue Handbook Offshore Steel Structures, Tapir Publishers, Norway, 1985.

[13] S. Haykin, Neural networks a comprehensive foundation, Prentice Hall, 2005.

[14] S. N. Londhe, S. Shah, P. R. Dixit, T. M. B. Nair, P. Sirisha, and R. Jain, "A Coupled Numerical and Artificial Neural Network Model for Improving Location Specific Wave Forecast," Applied Ocean Research, vol. 59, pp. 483-491, 2016.

[15] I. López, L. Aragonés, Y. Villacampa, and J. C. Serra, "Neural network for determining the characteristic points of the bars," Ocean Engineering, vol. 136, pp. 141-151, 2017.

[16] I. Lazakis, Y. Raptodimos, and T. Varelas, "Predicting ship machinery system condition through analytical reliability tools and artificial neural networks," Ocean Engineering, vol. 152, pp. 404-415, 2018.

[17] M. Smith, Neural Networks for Statistical Modeling, vol. 10, Van Nostrand Reinhold, 1993.

[18] M. T. Hagan, H. B. Demuth, M. H. Beale, and O. de Jesús, Neural Network Design, ISBN 0-9717321-0-8.

[19] Mathworks, "Neural Network Toolbox ${ }^{\mathrm{TM}}$ User's Guide, 2014". 
[20] S. F. Senra, Towards the Integration of Analysis and Design Methodologies of Marine Systems for Offshore Oil Exploitation [Dissertation, thesis], Department of Civil Engineering, COPPE/UFRJ, Rio de Janeiro, Brazil, 2004.

[21] DNV-RP-C203 (Det Norsk Veritas), Fatigue Design of Offshore Steel Structures, 2011. 


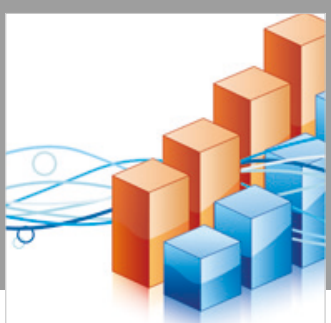

Advances in

Operations Research

\section{-n-m}
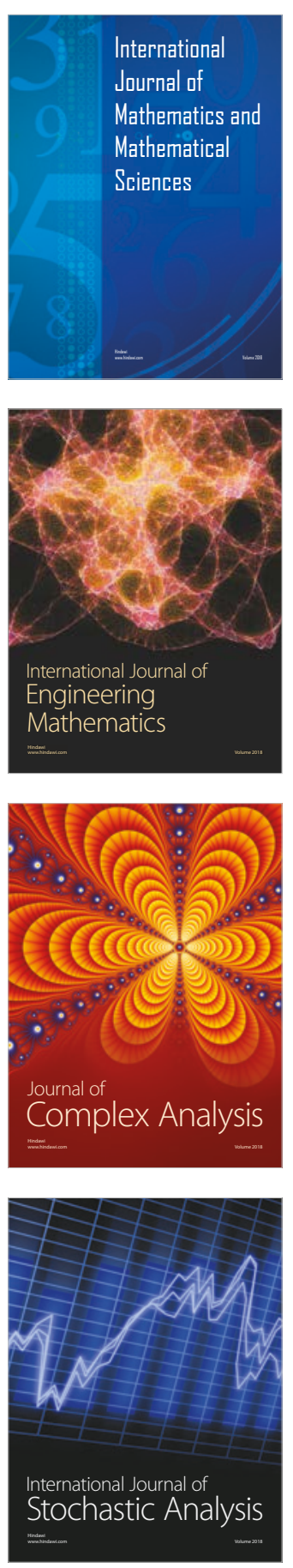
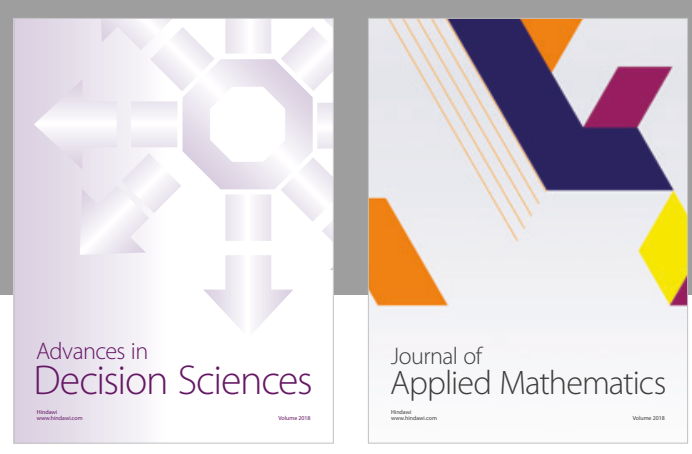

Journal of

Applied Mathematics
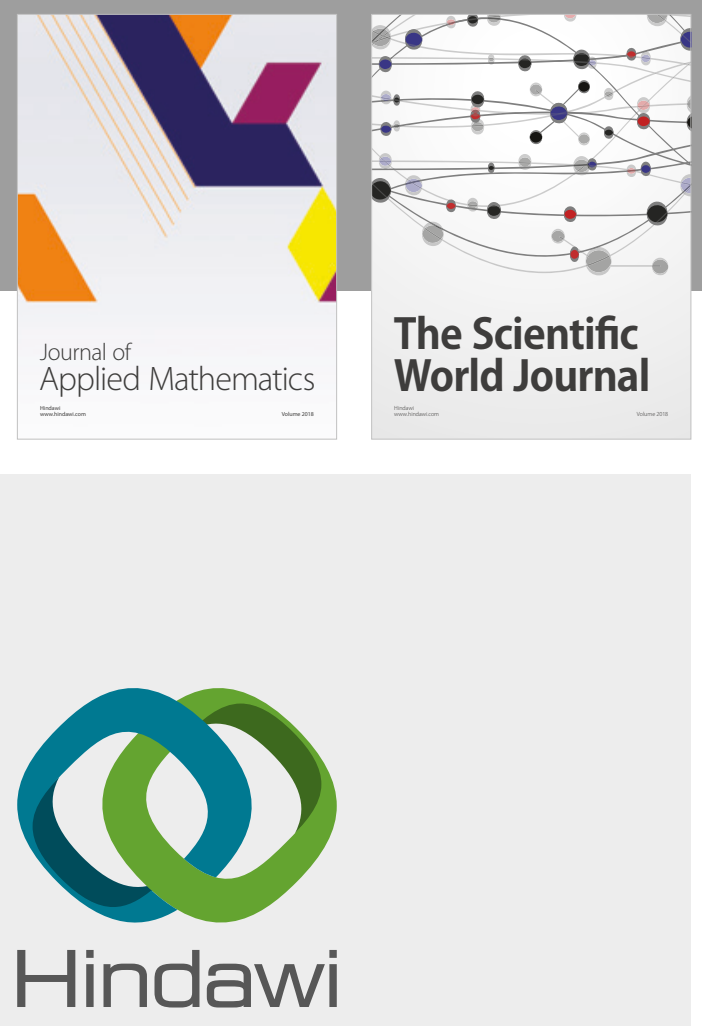

Submit your manuscripts at

www.hindawi.com

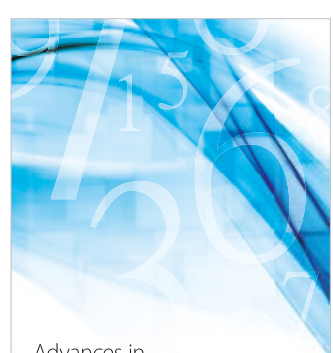

Advances in
Numerical Analysis
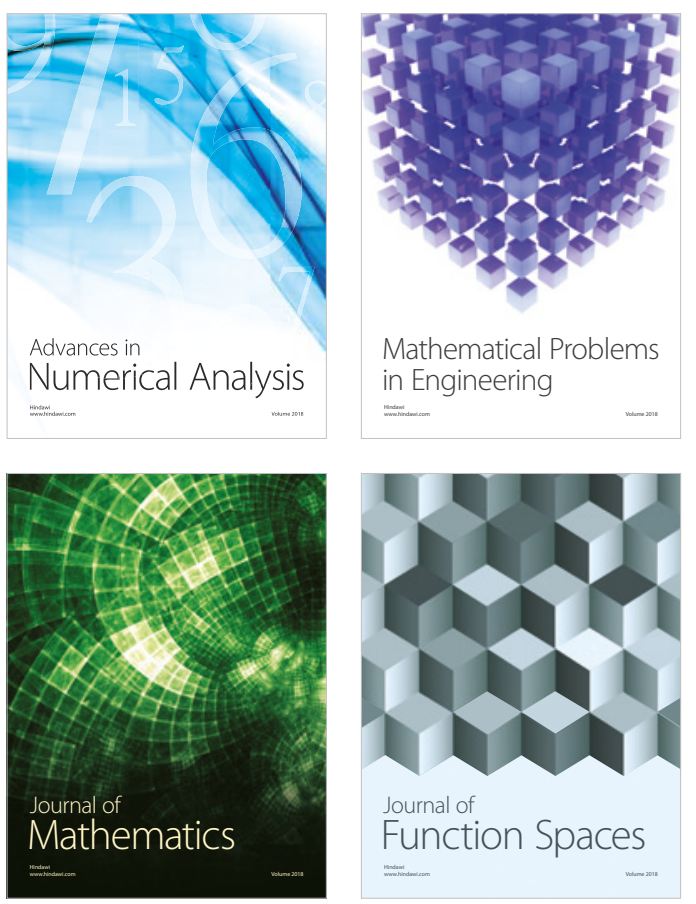

Mathematical Problems in Engineering

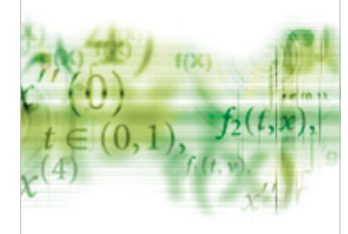

International Journal of

Differential Equations

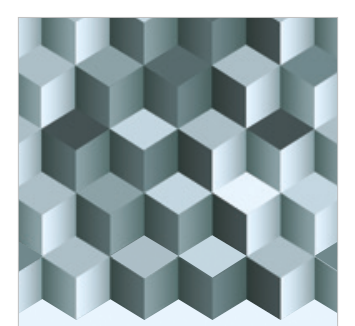

Journal of

Function Spaces

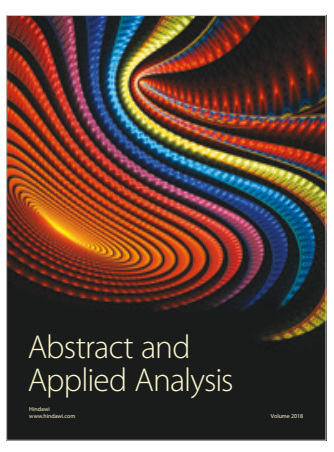

The Scientific

World Journal

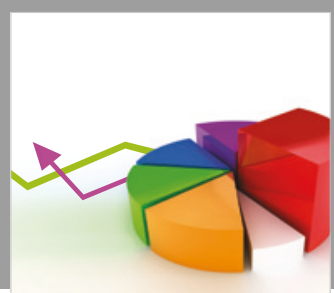

Journal of

Probability and Statistics
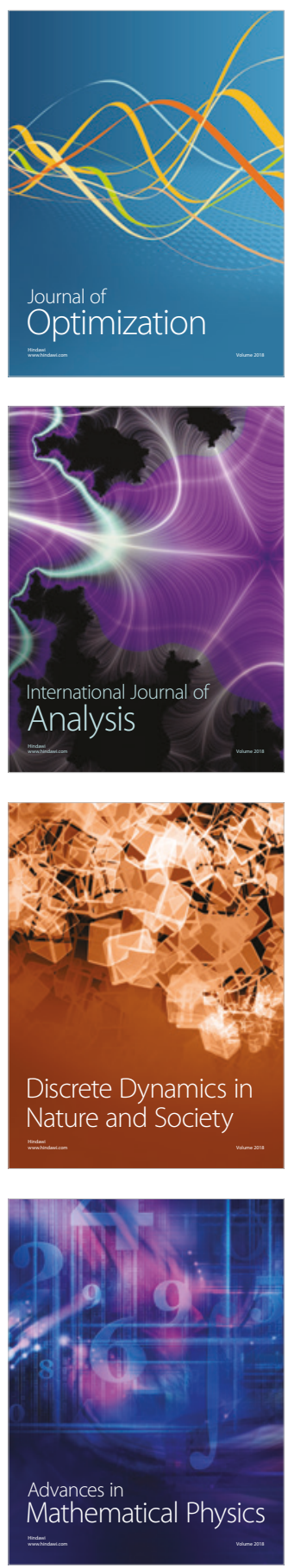